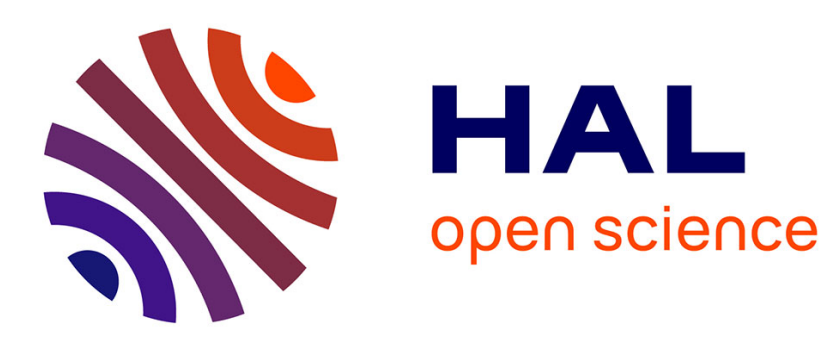

\title{
Evidencing the nonlinearity independence of resolution in femtosecond laser ablation
}

M. Garcia-Lechuga, O. Utéza, N. Sanner, Grojo D.

\section{To cite this version:}

M. Garcia-Lechuga, O. Utéza, N. Sanner, Grojo D.. Evidencing the nonlinearity independence of resolution in femtosecond laser ablation. Optics Letters, 2020, 10.1364/OL.382610 . hal-02490044

\section{HAL Id: hal-02490044 \\ https://hal.science/hal-02490044}

Submitted on 26 Feb 2020

HAL is a multi-disciplinary open access archive for the deposit and dissemination of scientific research documents, whether they are published or not. The documents may come from teaching and research institutions in France or abroad, or from public or private research centers.
L'archive ouverte pluridisciplinaire HAL, est destinée au dépôt et à la diffusion de documents scientifiques de niveau recherche, publiés ou non, émanant des établissements d'enseignement et de recherche français ou étrangers, des laboratoires publics ou privés. 


\title{
Optics Letters
}

\section{Evidencing the nonlinearity independence of resolution in femtosecond laser ablation}

\author{
M. Garcia-Lechuga, ${ }^{1, *}$ (1) O. Utéza, ${ }^{1}$ N. Sanner, ${ }^{1}$ and D. Grojo ${ }^{1,2}$ \\ ${ }^{1}$ Aix Marseille Université, CNRS, LP3, UMR7341, 13288 Marseille, France \\ ${ }^{2}$ e-mail: david.grojo@univ-amu.fr \\ *Corresponding author: garcialechuga@/p3.univ-mrs.fr.
}

Received 5 November 2019; revised 16 January 2020; accepted 17 January 2020; posted 17 January 2020 (Doc. ID 382610); published 12 February 2020

To overcome the resolution limits in laser processing technologies, it is highly attractive to translate concepts used in advanced optical microscopy. In this prospect, the nonlinear nature of absorption in dielectrics with femtosecond lasers is recurrently taken as a direct advantage in an analogous way to excitation in multiphoton microscopy. However, we establish that no direct benefit in resolution can be expected when laser ablation is observed. We explore widely different nonlinear regimes using ultrashort pulses at different wavelengths (1550 and $515 \mathrm{~nm}$ ) and target materials of various bandgaps $(3.8-8.8 \mathrm{eV})$. We find in the experiments that the shapes of all ablation features correspond to a one-to-one mapping of the beam contours at a strict threshold intensity. The nonlinearity-independent response shows that the incorporation of extreme UV should provide a direct route to the nanoscale resolutions routinely achieved in lithography. ( 2020 Optical Society of America

https://doi.org/10.1364/OL.382610

There are two unique advantages to using tightly focused femtosecond laser pulses for microfabrication technologies. First, the nonlinear nature of off-resonant ionization and energy deposition with intense light provides the ability to access threedimensional (3D) control in transparent materials, leading to major technological advances from 3D multiphoton lithography [1] to nanosurgery [2]. The second advantage is a process that becomes extraordinarily reproducible, because the energy thresholds for material responses move from stochastic for long pulses to deterministic for sub-picosecond pulses $[3,4]$. This has led to super-resolution machining demonstrations by tight control of the peak fluence, $F_{\text {peak }}$, slightly overpassing the threshold for material ablation, $F_{\text {th }}$. Joglekar et al. [5], by tightly focusing a femtosecond pulse at a $1053 \mathrm{~nm}$ wavelength on glass, were able to produce ablation features as small as $40 \mathrm{~nm}$ which is far below the diffraction limit $(<\lambda / 20)$.

Interestingly, similar sub-diffractive resolution performances have also been reported in the important field of multiphoton lithography [6]. By way of explanation, the concept of nonlinear resolution associated with an energy deposition profile confined in a region smaller than the beam has generally been evoked, as it is exploited in multiphoton microscopy [7]. A similar benefit is also assumed to contribute in surface ablation of transparent dielectrics [8-10].

Following this general concept of nonlinear resolution, and assuming for illustration energy deposition by pure multiphoton ionization (MPI), the absorbed energy profile is proportional to $[I(r)]^{n}$, with $I(r)$ being the intensity beam profile and $n$ being the number of photons required to span the bandgap of the material. Thus, for a Gaussian spot of waist $w_{\text {laser }}$, the size of the absorption profile, $w_{\text {MPI }}$, is reduced by a factor $1 / \sqrt{n}$. More rigorously, this factor may vary depending on the contribution of other absorption mechanisms, including tunneling and collision-assisted ionization. However, a resolution enhancement remains intuitively expected as long as a nonlinearity is associated to energy deposition.

In practice, the studies addressing the nonlinear resolution in laser ablation experiments are scarce. The main evidence was provided by Korte et al. [9], after obtaining a good correlation between the smallest achievable ablation features and the nonlinearity change by irradiating dielectrics with different bandgaps. However, the extremely tight focus in these experiments make very delicate the crater characterizations and fine comparisons of the morphologies required at same excitations levels (ratio $F_{\text {peak }} / F_{\text {th }}$ ) to undoubtedly evidence a benefit from nonlinear confinement.

Some other experiments with larger modifications feed the debate on this question. Puerto $e t a l$. [11] retrieved from analyses of ablation experiments at $800 \mathrm{~nm}$ a smaller spot size with fused silica (FS, $n=5)$ than the one derived with GeSbTe (GST, $n=1)$, but the reduction was very modest $\left(w_{\mathrm{FS}}=0.93 \cdot w_{\mathrm{GST}}\right)$ in comparison to expectations with a hypothetic pure MPI response $(1 / \sqrt{5} \approx 0.45)$. Recently, Sozet et al. [12] proposed a mono-shot methodology for damage threshold determination in dielectrics. It is based on imaging the incident laser beam (1 photon response) and single impact analysis on glass modification (nonlinear response). The good accuracy reported without accounting for any nonlinearity of absorption tends to contradict the concept of nonlinear resolution.

In this Letter, we focus on experiments unveiling this question, being very diligent in the experimental conditions of irradiation, in the analysis of the induced craters and in the characterization of the beam focal spot. This allows a reliable comparison between very different levels of nonlinearity 
changed by repeating the experiments for various materials and wavelengths. We demonstrate an independence of resolution to nonlinearity in femtosecond laser machining. More generally, the analyses highlight that there is a misconception in evoking together a nonlinearity-based resolution and a threshold response. While nonlinear energy deposition confines the absorbed energy profile, a strict fluence threshold response simply ruins all potential benefit on the resolution because of the subsequent binary experimental outcome independent of the interaction physics (e.g., ablated or non-ablated material depending on a local fluence).

To address experimentally the question, we have chosen highorder nonlinear absorption conditions. We perform single-shot irradiations on sapphire, $E_{g}=8.8 \mathrm{eV}$ [13], with pulses at a $1550 \mathrm{~nm}$ wavelength $\left(E_{p h}=0.8 \mathrm{eV}\right)$ and $190 \mathrm{fs}$ pulse duration at FWHM. Thus, the energy of 11 photons is required to cross the bandgap. The infrared pulses are delivered by an optical parametric amplifier pumped with pulses of $500 \mu \mathrm{J}$ at $1030 \mathrm{~nm}$ (Pharos, Light Conversion). An aspheric lens $(f=50-\mathrm{mm})$ focuses the beam onto the sample, which is mounted on XYZ motorized stages. Motion in the XY plane allows positioning of the sample on a fresh region from pulse to pulse. Micrometer precision Z-axis motion and in-situ surface microscopy imaging $\left(10 \times\right.$, tilted at $\left.45^{\circ}\right)$ ensures the positioning at the best focus. Afterwards, surface contaminations are cleaned with acetone, and craters are characterized by means of confocal microscopy (Leica DCM3D, $460 \mathrm{~nm}$ illumination, 150× objective lens), allowing us to measure the ablated area with sub-micrometer lateral precision.

Figure 1(a) shows the ablated area of the craters induced in sapphire after a single-shot irradiation at different pulse energies. The horizontal axis is in logarithmic scale for a comparison to Liu's methodology [14]. This methodology, based on the hypothesis of determinism when irradiating with ultrashort pulses, associates to the edge of a crater a local fluence equal to the ablation fluence threshold, $F_{\text {th }}$, being expressed for a Gaussian beam mathematically as

$$
F_{\text {th }}=F_{\text {peak }} \cdot e^{-2 R^{2} / w^{2}},
$$

where $R$ is the crater radius; $2 w$ is the beam spot diameter at $1 / e^{2}$ the peak value, and $F_{\text {peak }}$ is the peak fluence value, related with the pulse energy as $F_{\text {peak }}=2 E / \pi w^{2}$. This thresholding methodology can be transformed into a linear expression:

$$
A_{\text {crater }}=\frac{\pi w^{2}}{2} \ln \left(E / E_{\mathrm{th}}\right),
$$

with $A_{\text {crater }}\left(=\pi R^{2}\right)$ being the ablated area, and $E_{\text {th }}$ being the minimum pulse energy to induce material ablation $\left(F_{\mathrm{th}}=2 E_{\mathrm{th}} / \pi w^{2}\right)$. Thus, a linear regression applied to a set of measured ablated areas generated at different pulse energies allows us to retrieve $E_{\mathrm{th}}$ and $w$. In Fig. 1(a), the linearity between $A_{\text {crater }}$ and $\ln (E)$ is lost at energies above $40 \mu \mathrm{J}$ due to the deviation, as we will see later on, from a perfect Gaussian beam shape in our experiment. Ignoring this aspect, Liu's method applied for values below $40 \mu \mathrm{J}$ allows us to derive a pulse energy threshold for ablation, $E_{\mathrm{th}}=10.7 \pm 0.1 \mu \mathrm{J}$ (error according to fitting procedure).

The $1550 \mathrm{~nm}$ laser beam at the focal position is characterized with an imaging system composed of a microscope objective $(100 \times$, NA 0.5), a tube lens, and an InGaAs camera (Raptor
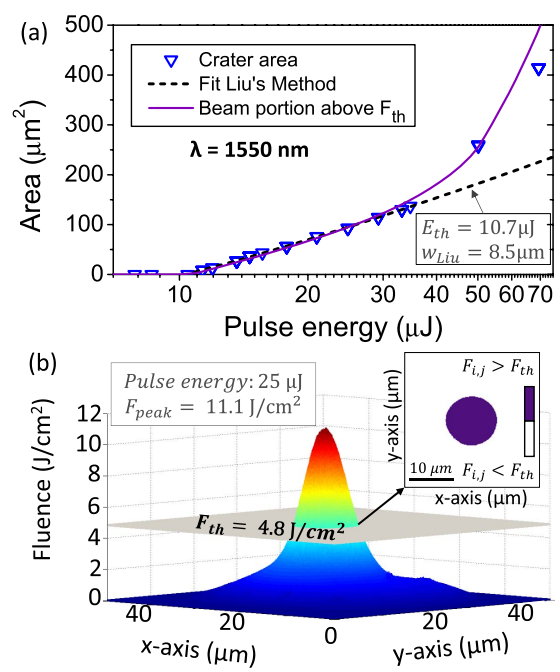

Fig. 1. (a) (Symbols) Ablated areas on sapphire after single-pulse irradiation $(\lambda=1550 \mathrm{~nm})$ as a function of the pulse energy. (Dashed line) Liu's method fitting the experimental values below $40 \mu \mathrm{J}$. (Solid line) Area of beam portions with local fluences above the fluence ablation threshold retrieved from the measured profile. (b) Fluence spatial distribution at the sample position for a $25 \mu \mathrm{J}$ beam as obtained using a calibrated infrared microscopy system. The inset displays the beam portion exhibiting values above the ablation fluence threshold.

OWL 640). The acquired 16-bit image, $I_{i, j}$ (a.u.), is translated in an absolute fluence distribution, $F_{i, j}\left(\mathrm{~J} / \mathrm{cm}^{2}\right)$, for a given incident energy, thanks to the linear response of the sensor and a calibration procedure. As an example, in Fig. 1(b), the fluence distribution for a pulse energy of $25 \mu \mathrm{J}$ corresponding to a peak fluence value equal to $F_{\text {peak }}=11.1 \mathrm{~J} / \mathrm{cm}^{2}$ is represented.

Therefore, the ablation fluence threshold corresponds to the peak fluence of a pulse with an energy equal to the energy threshold, obtaining for sapphire $F_{\text {th }}=4.8 \pm 0.5 \mathrm{~J} / \mathrm{cm}^{2}$. The $\pm 10 \%$ error here is an estimate taking into account the errors on beam imaging, considering spatial calibration uncertainties and background noise in the image. Despite this uncertainty, it is worth highlighting here that the fluence value obtained by beam imaging is more reliable than the one using the beam waist retrieved by Liu's method $\left(F_{\mathrm{th}}=2 E_{\mathrm{th}} / \pi w_{L i u}^{2}=9.4 \mathrm{~J} / \mathrm{cm}^{2}\right)$, since the real beam profile is exhibiting a pedestal deviating from the Gaussian profile assumed by Liu's method.

Of importance in this Letter is that the knowledge of the fluence distribution makes it possible to retrieve the beam portion exceeding the fluence ablation threshold $\left(F_{i, j}>F_{\mathrm{th}}\right)$. In Fig. 1(b), which corresponds to an excitation level equal to $F_{\text {peak }} / F_{\text {th }}=2.3$, it is shown in violet the portion of the beam with values above the threshold. This is obtained by simply thresholding (sectioning) the fluence profile with the plane at $F_{\text {th }}$. Following the same numerical thresholding strategy for different pulse energies, the curve represented in Fig. 1(a) is obtained. It is then observed that the size of the craters is directly corresponding to the area of beam portions above $F_{\mathrm{th}}$. Some differences are observed at high peak fluences, $\sim 7 \cdot F_{\text {th }}$, related to the limited dynamics of our infrared array sensor (thresholding at the pedestal level with a degraded signal-to-noise ratio).

In order to generalize this conclusion, we have repeated similar experiments in two different materials: fused silica infrasil 

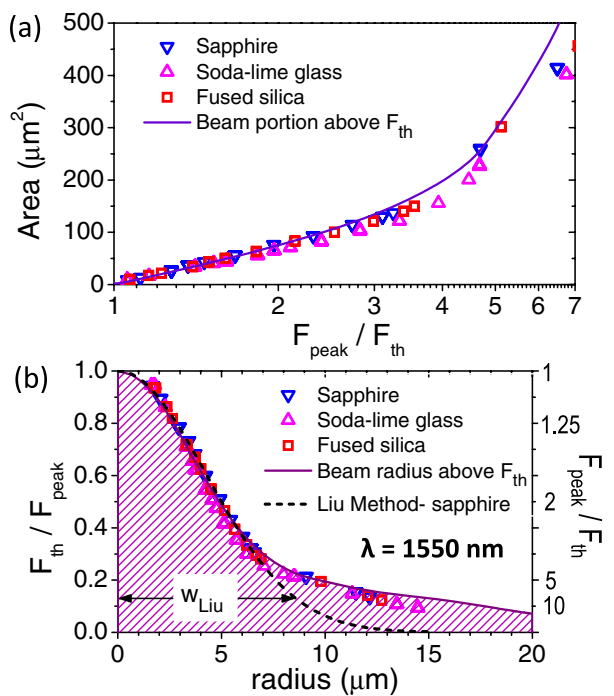

Fig. 2. (a) (Symbols) Ablated areas on different dielectrics after single-pulse irradiation $(\lambda=1550 \mathrm{~nm}, \Delta t=190 \mathrm{fs})$ as a function of a peak fluence normalized to ablation thresholds. (Solid line) Area of beam portions with fluences above the threshold retrieved from the measured profile. (b) Same data displayed after swapping axes in linear scales for a direct comparison of the dimension of the induced craters with the measured beam profile and a Gaussian approximation (Liu's methodology applied to sapphire data for $F_{\text {peak }}<4 \cdot F_{\text {th }}$.).

and a soda-lime glass with optical bandgaps of 5.2 and $3.8 \mathrm{eV}$ as measured by spectrophotometry. A relevant technical aspect here is the accuracy on the repositioning of the different samples at the best focal position which is guaranteed by exploiting the $10 \mu \mathrm{m}$ depth of field of the in-situ imaging system. The results on the three materials are plotted together in Fig. 2(a), representing the ablated area as a function of the normalized ratio $F_{\text {peak }} / F_{\text {th }}$, using the determined $F_{\text {th }}$ of each material. Additionally, a unique curve representing the measured beam portion above the fluence threshold is plotted, given that focusing conditions are the same. An alternative visualization is provided in Fig. 2(b), using the same data as in Fig. 2(a), but swapping the axes both now in linear scale and translating the area into an apparent radius $(R=\sqrt{\text { Area } / \pi})$. Under this representation, where the lateral dimension of the beam profile is plotted, it is confirmed that the size of the ablation feature is only a matter of thresholding at different ratios $F_{\text {peak }} / F_{\text {th }}$ the incident beam, a procedure totally independent of the nonlinear nature of light absorption.

The direct correlation between fluence profile thresholding and the induced craters is more visually expressed in Fig. 3. On the left side of the figure, we compare the beam portions with local fluences above $F_{\text {th }}$ and the laser-created craters in sapphire and soda-lime at three different excitation levels. One can note an excellent correlation between the contour shapes of craters and the beam spatial distributions. This observation confirms that the knowledge of the beam profile and fluence threshold is enough to predict the surface ablation in these conditions, regardless of the nonlinear order of absorption.

A first consequence for machining technologies is that the maximum resolution achievable can be evaluated by thresholding the beam profile close to the ablation fluence threshold, independently on the material. As shown on the left part in
Fig. 3 under the described focusing conditions, it is hard to induce modification below $2 \mu \mathrm{m}$ when slightly overcoming the ablation fluence threshold $\left(F_{\text {peak }}=1.07 \cdot F_{\text {th }}\right)$. Trying to achieve higher ablation resolution leads to hardly reproducible features as the difference between the targeted peak fluence, and the fluence threshold becomes on the order of typical pulse-to-pulse energy fluctuations ( $\sim 2 \%$ in our experiments).

Our results can also be exploited advantageously by expressing them inversely, as the dimensions of produced craters at different energy levels allow us to retrieve the laser beam shape, as shown in Fig. 2(b). This observation confirms that the simplified Liu's methodology [14] is also undoubtedly applicable when irradiating dielectrics, but limited to Gaussian beam shapes. For more complex beam shapes, a more general numerical procedure is required. Such an approach was successfully applied by Chalupský et al. [15] for spatial characterization of $\mathrm{X}$-ray ultrashort pulses through the ablative imprints produced in polymers $(n=1)$. Our results show that the approach is also valid for dielectric ablation under nonlinear absorption conditions.

However, it is worth mentioning here that not all materials seem equally appropriate for this purpose. Looking closer at the results on different materials, we see that slightly smaller ablated features (see Figs. 2 and 3) are observed in soda-lime. Interestingly, it is the lowest bandgap material tested in our experiments. This allows us to directly exclude a potential nonlinearity-based confinement of ablation, attributing the size reduction to a specific material response. Given the observation of elevations at the crater edge (see Fig. 3) and the borosilicate nature of soda-lime, we conclude on a similar hydrofluidic phenomenon as the one explained by Ben-Yakar et al. [16]. Therefore, close to the edges, the flow of molten material is partially refilling the peripheral regions leading to an apparent reduction of the ablated area in comparison to predictions assuming a threshold response based on local fluences. At the opposite, sapphire exhibits very neat craters (see Fig. 3) and can be consequently considered as a reference dielectric for impact-based beam characterization methods.

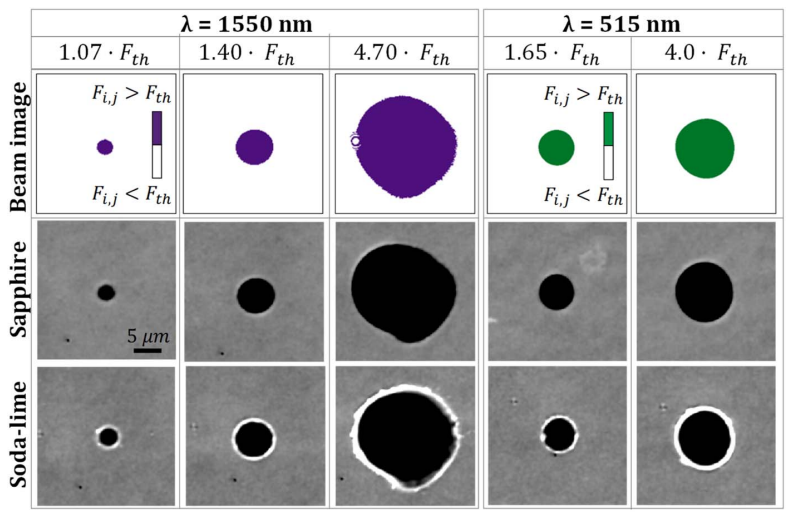

Fig. 3. (1st row) Beam portions above the ablation fluence threshold for different excitation levels $\left(F_{\text {peak }} / F_{\text {th }}\right)$. (2nd and 3rd rows) Confocal microscopy images of the surface modifications on sapphire and soda-lime induced at the indicated peak fluence above the ablation fluence threshold. Surface depressions and elevations are represented with darker and brighter colors, respectively. The three columns on the left correspond to the experiment performed at $1550 \mathrm{~nm}$ and the two columns on the right to the ones at $515 \mathrm{~nm}$. (Images size: $25 \mu \mathrm{m} \times 25 \mu \mathrm{m})$. 


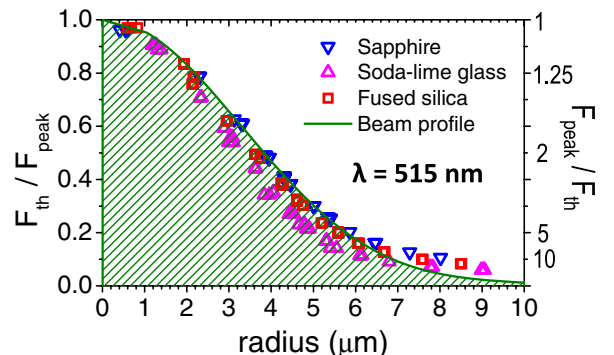

Fig. 4. Dimensions of ablated areas and the beam profile $(\lambda=515 \mathrm{~nm}, \Delta t=160 \mathrm{fs})$ extracted from the imaged beam at the focal position.

In order to generalize the conclusion on the absence of nonlinear resolution in femtosecond laser ablation, experiments have been carried out at a $515 \mathrm{~nm}$ wavelength $\left(E_{p h}=2.4 \mathrm{eV}\right)$ obtained through second-harmonic generation of the previously described source. The change of the wavelength gives another way to modify the nonlinear order, but also to change the balance between the expected nonlinear absorption mechanisms. The latter could represent another matter of debate if the conclusion were only valid at $1550 \mathrm{~nm}$. This is because a long wavelength favours tunneling in comparison to MPI, and an electron avalanche efficiently develops [17]. Experiments at $515 \mathrm{~nm}$ surely change the balance between these mechanisms giving more importance to the MPI response.

Following the same representation as in Fig. 2(b), we compare in Fig. 4 the lateral dimensions of the imaged beam profile and craters induced in the three different samples. Taken together with the images shown on the right part of Fig. 3, this again confirms that the size and contour of craters can be predicted by simple thresholding of the beam profile, independently of the nonlinear process responsible for light absorption. Additionally, in Fig. 3, it is shown that even irradiating at radically different nonlinear regimes, similar crater morphologies are induced on both materials: neat modification in sapphire and elevations and inhomogeneous edges in soda-lime. This is consistent with our interpretations of the detailed features based on the material responses.

In summary, we have investigated the femtosecond laser ablation response for different nonlinear excitation regimes, varying the apparent multiphoton order of absorption from 2 to 11 . We found that the ablated areas correspond systematically to exact imprints of the measured beam profile at a strict threshold fluence. This demonstrates the absence of nonlinear resolution in femtosecond laser ablation, unraveling the contradiction in evoking together the nonlinearity and intensity-threshold response for resolution enhancement.

This is a misconception that has emerged since the advent of femtosecond laser processing and still persists today, because nonlinear energy deposition is analogous to excitation in multiphoton microscopy. However, one should not ignore that a major difference in this comparison is in the subsequent response. In microscopy, a progressive fluorescence response follows the nonlinear confinement of absorption. However, a threshold-based material response (Heaviside step response) will lead to an observation that depends only on whether the locally delivered fluence is below or above a threshold, without any accessible benefit on resolution from the nonlinear confinement of absorption.
While we have concentrated the experimental work on surface ablation for direct visual evidences, the conclusion holds for any threshold-based material transformations as photopolymerization or refractive index modifications associated to structural and morphological changes. These are the bases of various surface and $3 \mathrm{D}$ technologies, as multiphoton lithography or waveguide writing, for which the contributions of nonlinear confinement and thresholding to resolution are regularly mentioned together $[1,18]$. By showing their incompatibility, we clarify an important aspect for more resolution advances.

Finally, it is striking to note that the best resolutions in ultrashort laser ablation were demonstrated with femtosecond lasers emitting in the near-infrared [5]. This part of the spectrum is highly favourable for efficient coupling to materials, but obviously is not an optimum in dealing with diffraction limits. Therefore, we consider that, following a trend similar to lithography [19], efforts should be devoted to incorporating tightly focused extreme UV ultrashort pulses (e.g., high-harmonic generation) in the field of surface laser machining or modification. This must open a direct route to nanoscale control that would allow nanosurgery of cells or of DNA and to tailor solid surfaces with unprecedented resolution.

Funding. H2020 European Research Council (724480).

Acknowledgment. M. Garcia-Lechuga is grateful to Dr. Jörn Bonse for fruitful discussions and advices.

Disclosures. The authors declare no conflicts of interest.

\section{REFERENCES}

1. S. Kawata, H.-B. Sun, T. Tanaka, and K. Takada, Nature 412, 697 (2001).

2. M. F. Yanik, H. Cinar, H. N. Cinar, A. D. Chisholm, Y. Jin, and A. BenYakar, Nature 432, 822 (2004).

3. D. Du, X. Liu, G. Korn, J. Squier, and G. Mourou, Appl. Phys. Lett. 64, 3071 (1994).

4. N. Sanner, O. Utéza, B. Chimier, M. Sentis, P. Lassonde, F. Légaré, and J. C. Kieffer, Appl. Phys. Lett. 96, 071111 (2010).

5. A. P. Joglekar, H.-H. Liu, E. Meyhofer, G. Mourou, and A. J. Hunt, Proc. Natl. Acad. Sci. 101, 5856 (2004)

6. N. Li, R. R. Gattass, E. Gershgoren, H. Hwang, and J. T. Fourkas, Science 324, 910 (2009).

7. W. R. Zipfel, R. M. Williams, and W. W. Webb, Nat. Biotechnol. 21, 1369 (2003).

8. M. Lenzner, Int. J. Mod. Phys. B 13, 1559 (1999).

9. F. Korte, J. Serbin, J. Koch, A. Egbert, C. Fallnich, A. Ostendorf, and B. N. Chichkov, Appl. Phys. A 77, 229 (2003).

10. K. Sugioka and Y. Cheng, Appl. Phys. Rev. 1, 041303 (2014).

11. D. Puerto, W. Gawelda, J. Siegel, J. Solis, and J. Bonse, Appl. Phys. Lett. 92, 219901 (2008).

12. M. Sozet, J. Neauport, E. Lavastre, N. Roquin, L. Gallais, and L. Lamaignère, Opt. Lett. 41, 804 (2016).

13. R. H. French, J. Am. Ceram. Soc. 73, 477 (1990).

14. J. M. Liu, Opt. Lett. 7, 196 (1982).

15. J. Chalupský, J. Krzywinski, L. Juha, V. Hájková, J. Cihelka, T. Burian, L. Vyšín, J. Gaudin, A. Gleeson, M. Jurek, A. R. Khorsand, D. Klinger, H. Wabnitz, R. Sobierajski, M. Störmer, K. Tiedtke, and S. Toleikis, Opt. Express 18, 27836 (2010).

16. A. Ben-Yakar, R. L. Byer, A. Harkin, J. Ashmore, H. A. Stone, M. Shen, and E. Mazur, Appl. Phys. Lett. 83, 3030 (2003)

17. L. V. Keldysh, Sov. Phys. JETP 20, 1307 (1965).

18. G. Zhang, G. Cheng, M. K. Bhuyan, C. D’Amico, Y. Wang, and R. Stoian, Photonics Res. 7, 806 (2019).

19. C. Wagner and N. Harned, Nat. Photonics 4, 24 (2010). 